\title{
Monitoring of residential drinking water service consumption using Human Machine Interaction $(\mathrm{HCl})$ techniques
}

\author{
David Chilcañán \\ Departamento de Eléctrica y \\ Electrónica \\ Universidad de las Fuerzas Armadas \\ ESPE \\ Av. General Rumiñahui s/n 171-5- \\ 231B Sangolquí, Ecuador \\ dachilcannc@espe.edu.ec
}

\author{
Cristopher Cuzco \\ Departamento de Eléctrica y \\ Electrónica \\ Universidad de las Fuerzas Armadas \\ ESPE \\ Av. General Rumiñahui s/n 171-5- \\ 231B Sangolquí, Ecuador \\ cacuzco@espe.edu.ec
}

\author{
Alvaro Uyaguari \\ Departamento de Eléctrica y \\ Electrónica \\ Universidad de las Fuerzas Armadas \\ ESPE \\ Av. General Rumiñahui s/n 171-5- \\ 231B Sangolquí, Ecuador \\ aduyaguari@espe.edu.ec
}

\begin{abstract}
The integration of the drinking water network with information and communication technologies (ICTs) is the future of automated metering for users and service providers, the modern world cannot be conceived without water and the current infrastructure of manual metering, is poorly adapted to current needs, every day the demand for this vital liquid is greater and data collection from meters must be automated, allowing users to make queries of water consumption and tariffs to pay, however, it is necessary to create a centralized intelligent network with better adaptability to the meter; Implementing these technologies is expected to significantly reduce reading times in measurements, failure reports and changes in the drinking water network. In developing countries such as Ecuador, does not provide a metering infrastructure, however in the country, is thinking about creating Smart Grids for the adequacy of intelligent cities, a big step into the future.

This article presents an implementation proposal for Smart Water Meter (SWM), by means of a virtual assistant and a metering network, allowing to consult the $\mathrm{m} 3$ of water consumed per day, week and month, as well as the total amount to be paid per consumption, integrating hardware and free software, for greater security in communications.
\end{abstract}

\section{CCS Concepts}

- Information System Management Database. • Omnipresent Technology $\rightarrow$ Interconnected electronic devices. • Mediation Logic $\rightarrow$ Communication between applications

\section{Keywords}

Virtual Assistant, Chatbot, IoT, Natural language processing, Instant Messaging, Telegram, Smart Meters, ESP8266, HumanMachine(H2M), Machine-Machine(M2M).

\section{INTRODUCTION}

Currently, several countries around the world tend to grow exponentially in population, generating a great influence on the economic and social development of the country. Mainly the cities of great population affluence are those that generate an environment where people live, work and consume more and more resources. This complex ecosystem allows in a more technical way to transform the management models commonly

ICAAI 2019, October 26-28, 2019, Istanbul, Turkey.

Copyright 2010 ACM 1-58113-000-0/00/0010 _..\$15.00.

DOI: http://dx.doi.org/10.1145/12345.67890 used, giving rise to the concept of Smart City, or city that makes use of information and communication technologies (ICTs) so that its infrastructure and the services provided are managed autonomously and efficiently[1]. Innumerable advantages have been attributed to this process, from the reduction of public spending to the automation of processes, allowing domestic water to be managed safely and efficiently.

In this context, the main challenges involved in the implementation of a measurement and control system, with emphasis on a comparison of functionalities and technical characteristics of intelligent meters used in the international market. Precisely the intelligent metering is a priority aspect for users, defining this as an intelligent network, allowing a HumanMachine (H2M) and Machine-Machine (M2M) communication, creating an advanced metering infrastructure (AMI)[2], integrating intelligent meter systems, communication networks and data management systems allowing two-way communication between public-private service companies with customers[3][4].

As a characteristic element of an Intelligent system, is born the Internet of Things (IoT), a digital interconnection of everyday objects with the Internet[5], adapting the prototype developed to the 7 layers of the IoT[6], creating an intelligent network with secure communication protocols (HTTPS - MQTT), improving interaction with users through a virtual assistant.

The article has been organized as follows: Section II describes the background and related works that support this research, section III implementation, section IV shows the results and discussions and finally, section $\mathrm{V}$ establishes conclusions based on the results obtained and describes future work.

\section{BACKGROUND AND RELATED WORKS}

\subsection{Smart Water Meters}

Smart metering is strongly related to the concept of Smart Grids, creating an intelligent network infrastructure for the reception and emission of data to suppliers and users, with one of the main objectives being to raise awareness of the efficiency of natural resources[7][8], economizing resources and saving time in data collection and informing users of consumption and the values to be paid, per $\mathrm{m}^{3}$ consumed. Although smart water metering is a practice recently implemented in developed countries, there are 
smart water meters on the market. However, in Ecuador there is no such service, using manual metering.

Despite the great advances in intelligent automatic metering has shown great efficiency for reducing excessive consumption and for timely detection of faults, however today service providers have focused on energy and gas consumption, leaving aside the residential drinking water service. The growing interest in sustainability in modern society has stimulated and fostered the development of the market for intelligent water metering to combat water leaks[9][10].

\subsection{Conversational Bots or ChatBots}

Software applications that interact in complex virtual environments where they execute tasks automatically, communicating with human characteristics, displaying text, graphics, icons, voice, animations, multimedia or virtual reality[11]. The main objective of Conversational Agents or Chatbots is to establish a dialogue with other interlocutors, either as a customer service, answering machines or for users to acquire information or receive instructions [12].

Chatbots currently use computational linguistics and Artificial Intelligence (AI) techniques to process natural language in order to understand or interpret what the user is trying to express. However, to simplify the development process, they are usually adapted to systems based on rules (questions and answers), and in other cases with reasoning based on cases. Therefore, they are programmed and designed mono or multitask, improving humanmachine communication (HCI)[13][14].

Understanding the functioning of Chatbots, it can be seen that a new communication channel is created with users, abandoning emails, web applications, voice communication, etc.. In addition, the speed of response of Chatbots increases user satisfaction, generating a feeling of constant attention, a service that users value positively.

\subsection{Computational Linguistics and Artificial Intelligence (AI).}

It is an interdisciplinary work that incorporates the handling of human language in computers, however it is directly associated with Artificial Intelligence (AI) for decision making, using natural language processing (NLP). Its objective is to help machines understand and decipher human language, creating effective communication. Artificial Intelligence (AI) manipulates information that is much more symbolic than numerical. The Information with which AI operates is more related to objects, facts, concepts and rules; in such a way that it resembles the reasoning usually used by human beings[15].

\subsubsection{Natural Language Processing (NLP)}

It is the ability of communication between man-machine or machine-machine using natural human language. In order to understand in detail, the syntax of sentences must be analyzed, interpreted in a semantic way and a structure based on knowledge must be created [16].

Natural language processing includes different techniques for interpreting human language, ranging from statistical and machine-based learning methods to rule-based and algorithmic approaches. The implementation of these systems requires a wide variety of methods, because text-based and voice-based data vary widely, as do practical applications [17][11]. In order for the computer to better understand human language, it is necessary to resort to different areas of linguistics[18]:
Morphology deals with the composition of words and their relationships with other words.

Syntax defines how words are joined to form phrases.

Semantics refers to both the meaning of words and groups of words.

Pragmatics echoes the context in which linguistic locutions are carried out.

Phonology deals with the phonetic structure of spoken language and is important for voice recognition.

In all these cases, the main objective is to take the raw text of the language and apply linguistics and AI algorithms or mathematical models to transform and enrich the text in such a way as to provide greater value and improve communication.

\subsection{Internet of Things (IoT)}

It is a concept that refers to the digital interconnection of devices with Internet, these devices have a unique identifier and the ability to transfer data through the network, allowing to interact independently with any other individual, either a MachineMachine communication (M2M) or Human-Machine (M2H) [19]. These devices can be controlled remotely inside or outside the network.

\subsection{Advanced Measurement Infrastructure (AMI)}

Intelligent Metering Infrastructure (AMI) is an application of wireless sensor networks (WSN) that include intelligent sensors or meters (SM), various communication systems that allow the monitoring, control and charging of the use of different types of services: electricity, domestic gas, drinking water and others[20][21], facilitating remote reading (users-drinking water company), automatic disconnection and reconnection (drinking water company), rapid replacement of service in contingencies, implementation of prepaid service which translates into a significant improvement in the quality of service to end users. Thus, in order to guarantee the collection of information, resources must be used optimally in order to minimize costs. In this way, reusing technologies allows us to reduce the costs of a new investment, among which we can include cellular networks and others of lesser scope focused on intelligent networks such as IEEE802.15.4 g, as opposed to wired technologies that due to the limitations of scope and the complexity of implementation in areas that are difficult to access [22].

\subsection{Related Works}

There are some works related to the Internet of Things (IoT) and the support it provides to new technologies, allowing the exchange of information, communicating not only People to Machine but also Machine to Machine (M2M), through the appropriate use of energy resources, through message-oriented middleware for intelligent networks. However, at present, this field of research has allowed the creation of several applications, which are integrated to remotely controlled devices through the Internet or wireless networks, providing benefits for agriculture, transport, industry, home, etc., however The monitoring of these systems is limited, and does not produce concrete results and easy interpretation. 


\section{IMPLEMENTATION}

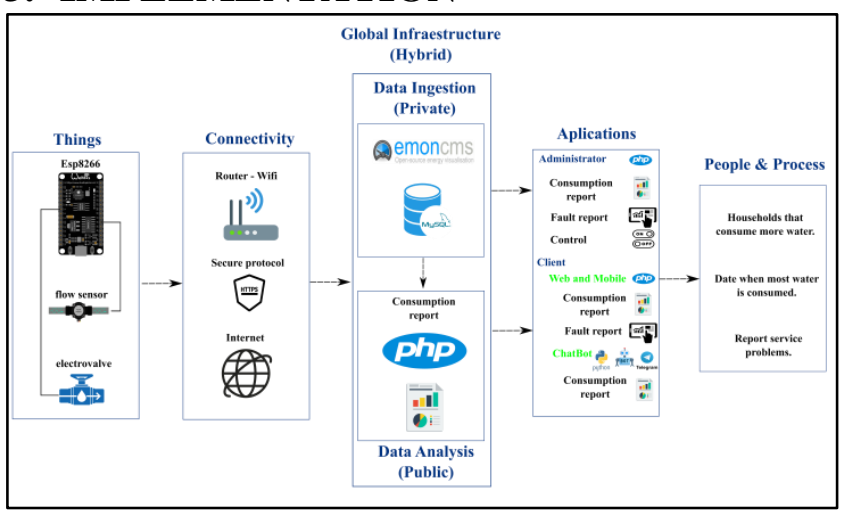

Figure 1: Functionality architecture based on the 7 IoT layers.

The architecture chosen for the development of this research allowed from a global approach to decrease response times, create a reliable operation between the proposed modules and interoperability between hardware to support different characteristics depending on the scenario, as shown in Figure 1.

\subsection{Things}

The connected devices (hardware) for the measurement fulfill specific functions for the intelligent metering (software), creating a meter that adapts to the needs of users and the company distributing drinking water, functioning as a receiver and transmitter of data on water consumption in $\mathrm{m}^{3}$. In addition, the meter can be controlled remotely from the company, allowing the flow of water to be cut off if the respective consumption balance has not been paid. The ESP8266 board creates an access point to the device and its components through a WIFI connection, allowing the data obtained from a flow sensor to be transmitted, and controlling the flow of water through an electrovalve. The smart meter and all its components operate at an average voltage of $12 \mathrm{~V}$, allowing the device to operate correctly as shown in Figure 1.

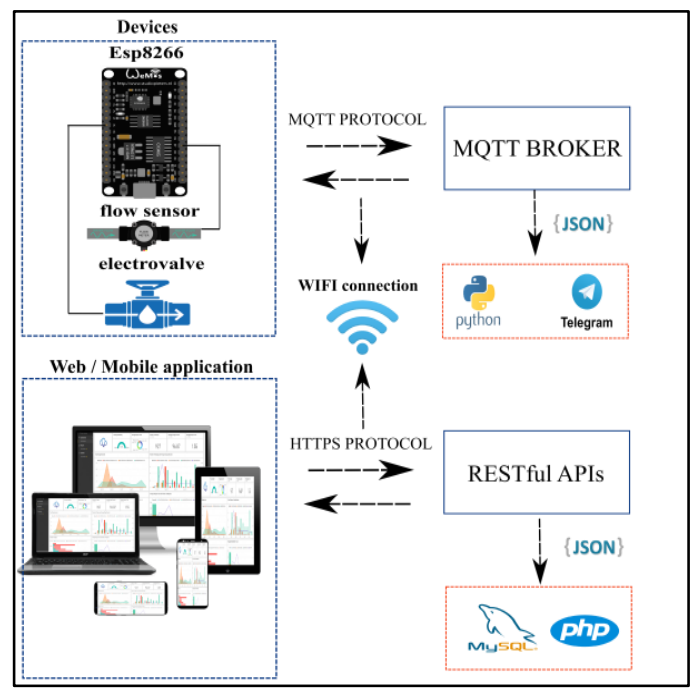

Figure 2: Secure communication and connectivity infrastructure.

\subsection{Connectivity}

The WIFI connection allows the device to create an access point to the device through a secure communication HTTPS (Hypertext
Transfer Protocol Secure) and MQTT (Message Queuing Telemetry Transport) for sending data to a dedicated front end and to the virtual assistant for user interaction and a dedicated front end of the water distribution company, as shown in Figure 2.

\subsection{Data Ingestion(Private)}

Real-time drinking water consumption can be viewed on Emoncms, an open-source web platform from OpenEnergyMonitor OEM, which allows you to integrate data sent from ESP8266 on water consumption in $\mathrm{m}^{3}$, over the course of the day, week or month, while the device is on. The consumption will also be stored every 30 minutes in a MySQL database on an Apache web server, so that the necessary data can be extracted to show it to the user and to the drinking water company.

\subsection{Data Analysis(Public)}

Once the data has been stored, the necessary fields can be extracted (consumption in $\mathrm{m}^{3}$, value to be paid per month, dates of consumption per day, week and month), identifying the day that consumes the most drinking water in the home, these data will be shown in the front-end written in php on a server dedicated to users, and on another server for the drinking water company, allowing a better detail of the consumption in $\mathrm{m}^{3}$, of the values to be charged, and above all the possibility of making cuts remotely through the front end of the company.

\subsection{Aplications (Web and mobile)}

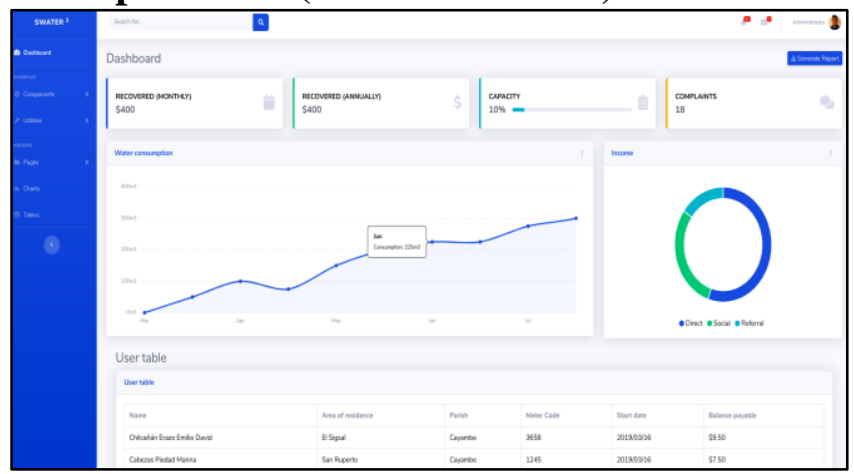

Figure 3: Front-end of the manager of the drinking water company.

As visualized in Figure 3, the administrator of the water distribution company will be able to dynamically visualize the consumption per $\mathrm{m}^{3}$ and the values to be paid by the user connected to the intelligent metering infrastructure, so that the data are objective, without the possibility of alterations. By means of this front end it will be possible to verify the data of the owner of each meter, reports of failures, values collected monthly and annually, and above all to carry out the cut of the service remotely, and to the contrary. The data obtained from the sensors in Figure 2, will be reflected on the website, creating dynamic and intuitive graphs, visualizing consumption statistics, values receivable and complaints made.

To enter the front-end of the users, it is necessary the meter number, so that you can view their personal data, report service failures, values collected monthly and annually, making it easier for users to see the actual consumption in $\mathrm{m}^{3}$ and the value to pay. The virtual assistant (chatbot) plays a fundamental role in notifying the user's Smartphone of the water consumption, and of the values to be paid, in such a way that the system interacts with 
the human, by means of natural language processing techniques, creating a Human-Machine communication, as shown in Figure 4.

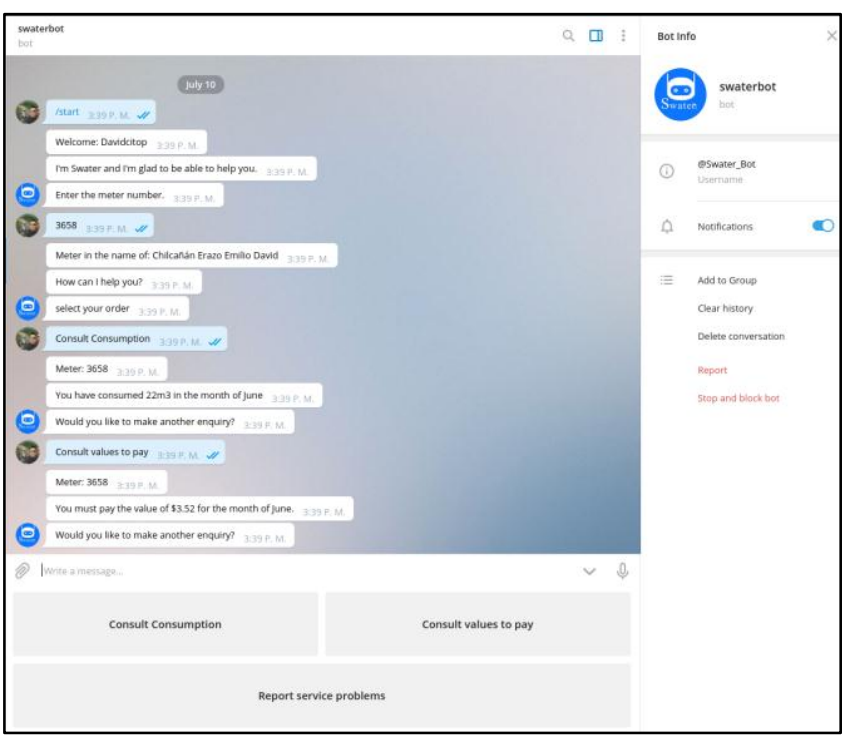

Figure 4: Operation of the virtual assistant (chatbot).

The virtual assistant (chatbot) is available 24 hours a day, 7 days a week, interacting through text messages applying natural language processing (NLP), for example (How much is the water consumption?) or preset commands (/ConsultConsumption). The data obtained by the sensors in Figure 2 are stored in a relational database, allowing queries through API REST, obtaining a .json with the requested data and presenting users dynamically through the virtual assistant and the website.

\section{RESULTS AND DISCUSSIONS}

As a case study and validation of the functioning of the smart meter (SM), tests were carried out in a real environment, installing the prototype in two houses in the City of Cayambe, during 4 months (March, April, May, June) as shown in Figure 5. The data sent from ESP8266 to the server are in $\mathrm{m}^{3}$ and the value to pay for drinking water consumption is monthly (30 days). Taking into account the table of prices per $\mathrm{m}^{3}$.

Table 1. Values established by the drinking water company (m3).

\begin{tabular}{|c|c|}
\hline \multicolumn{2}{|c|}{ Consumption Ranges } \\
\hline Consumption (m3) & Price $(\$)$ \\
\hline $0-15$ & 0.10 \\
\hline $15-30$ & 0.16 \\
\hline $30-60$ & 0.20 \\
\hline $60-120$ & 0.22 \\
\hline$>120$ & 0.26 \\
\hline
\end{tabular}

Applying the following formula, the consumption in $\mathrm{m}^{3}$ was checked against the actual values to be paid.

(House 1)

$$
\mathrm{Vtp}=\mathrm{Cs} * 0.16
$$

(House 2)

$$
\mathrm{Vtp}=\mathrm{Cs} * 0.10
$$

Where every letter means:

Vtp: Total value payable

Cs: Consumption in $\mathrm{m} 3$

Table 2.Monthly payment values per $\mathrm{m3}$.

\begin{tabular}{|l|l|l|l|l|}
\hline \multicolumn{5}{|c|}{ Consumption Ranges } \\
\hline \multirow{3}{*}{ Month } & \multicolumn{2}{|c|}{ Consumption (m) } & \multicolumn{2}{c|}{ Price $\$$ ) } \\
\cline { 2 - 5 } March & House 1 & House 2 & House 1 & House 2 \\
\cline { 3 - 5 } & 18 & 1 & 2.88 & 0.1 \\
\hline April & 15 & 13 & 2.40 & 1.30 \\
\hline May & 20 & 12 & 3.20 & 1.20 \\
\hline June & 22 & 11 & 3.52 & 1.10 \\
\hline
\end{tabular}

Based on the data obtained (House 2) from Table 2, the corresponding graph of drinking water consumption was made as shown in Figure 5.

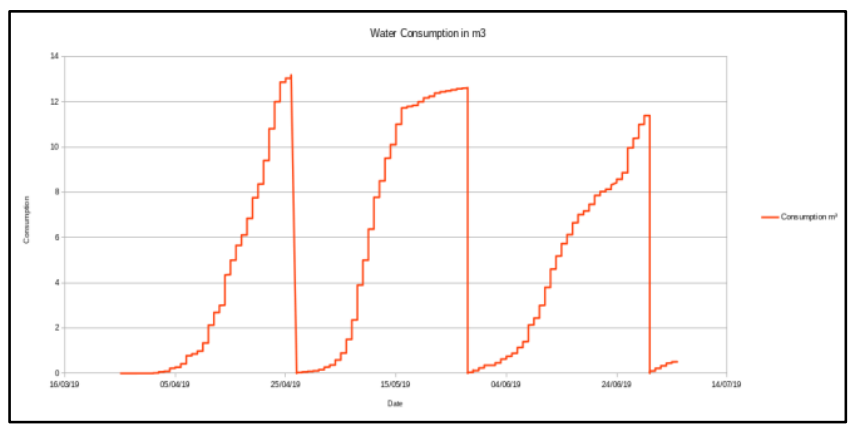

Figure 5: Monthly consumption of drinking water in $\mathrm{m} 3$ per month (House 2).

Based on the calculated data, the correct functioning in a real environment was verified during 4 months of testing, facilitating the reading of drinking water to the company as well as to the users, concluding that the average consumption of residential drinking water in the City of Cayambe is $14 \mathrm{~m} 3$ during 1 month.

As can be seen, the smart meter (SM) works correctly in a real environment, deducing that the data obtained has a margin of error of $1 \%$ as shown in Figure 5. Having these parameters available, it can be deduced that intelligent metering works and adapts to users' needs, avoiding excessive payment rates, measurement problems and saving economic resources, integrating Research, Development and Innovation $(\mathrm{R}+\mathrm{D}+\mathrm{i})$ to improve users' daily lives, providing technological solutions to society's problems at a local and international level.

\section{CONCLUSIONS AND FUTURE WORKS}

The intelligent meter automated the process of measuring drinking water consumption, making it easier for users and the drinking water company to interact with the system, allowing them to consult consumption in $\mathrm{m}^{3}$ and the values to be paid. This allowed the creation of an intelligent metering infrastructure with data transparency, fostering an open data culture.

The processing of the natural language of the proposed virtual assistant (chatbot), facilitated a more fluid communication with 
the users, allowing to send orders to consult the consumption in $\mathrm{m}^{3}$, the values to be paid and to report failures in the service.

The result is adapted according to the sector or country where the tests are carried out, since the price per $\mathrm{m} 3$ will have a different cost.

The tools used in the research facilitated communication between the user and the machine, covering a wide field of research on human-machine interaction.

It is proposed as a future work to incorporate the virtual assistant, a neuronal network that facilitates the automatic learning of the interaction with the users, allowing to incorporate a single meter of water and electrical current of the houses, obtaining a record in real time of both service, maintaining a Human-Machine communication, classifying and storing in a database, orders, conversations and IoT commands.

\section{REFERENCES}

[1] Y. Nait Malek, A. Kharbouch, H. El Khoukhi, M. Bakhouya, V. De Florio, D. El Ouadghiri, S. Latre, C. Blondia, On the use of IoT and Big Data Technologies for Real-time Monitoring and Data Processing, In Procedia Computer Science, Volume 113, 2017, Pages 429-434, ISSN 18770509

[2] Tram, H. (2010, April). Advanced metering infrastructure as an enabler of demand response. In IEEE PES T\&D 2010 (pp. 1-1). IEEE.

[3] Mustafa Alper Akkaş, Radosveta Sokullu, An IoT-based greenhouse monitoring system with Micaz motes, Procedia Computer Science, Volume 113, 2017, Pages 603-608, ISSN 1877-0509

[4] Michele Albano, Luis Lino Ferreira, Luís Miguel Pinho, Abdel Rahman Alkhawaja, Message-oriented middleware for smart grids, Computer Standards \& Interfaces, Volume 38, 2015, Pages 133-143, ISSN 0920-5489

[5] Chilcañán, D., Navas, P., \& Escobar, S. M. (2017, June). Expert system for remote process automation in multiplatform servers, through human machine conversation. In Information Systems and Technologies (CISTI), 2017 12th Iberian Conference on (pp. 1-8). IEEE.

[6] http://www.iotsense.io/blog/the-layers-of-iot/.

[7] Santos J., Vanhove T., Sebrechts M., Dupont T., Kerckhove W., Braem B., Van Seghbroeck G., Wauters T., Leroux P., Latre S., et al. City of Things: Enabling Resource Provisioning in Smart Cities. IEEE Commun. Mag. 2018;56:177-183. doi: 10.1109/MCOM.2018.1701322.

[8] Willis R.M., Stewart R.A., Panuwatwanich K., Williams P.R., Hollingsworth A.L. Quantifying the influence of environmental and water conservation attitudes on household end use water consumption. J. Environ. Manag. 2011;92:1996-2009. doi: 10.1016/j.jenvman.2011.03.023.

[9] Britton T.C., Stewart R.A., O'Halloran K.R. Smart metering: Enabler for rapid and effective post meter leakage identification and water loss management. J. Clean. Prod. 2013;54:166-176. doi: 10.1016/j.jclepro.2013.05.018.
[10] Loureiro D., Vieira P., Makropoulos C., Kossieris P., Ribeiro R., Barateiro J., Katsiri E. Smart metering use cases to increase water and energy efficiency in water supply systems. Water Sci. Technol. 2014;14:898-908. doi: 10.2166/ws.2014.049.

[11] Chilcañán, D., Navas, P., \& Escobar, M. (2018, July). Virtual Assistant for IoT process management, using a middleware. In Proceedings of the 2018 2nd International Conference on Algorithms, Computing and Systems (pp. 209-213). ACM.

[12] Jennifer Hill, W. Randolph Ford, Ingrid G. Farreras, Real conversations with artificial intelligence: A comparison between human-human online conversations and humanchatbot conversations, Computers in Human Behavior, Volume 49, 2015, Pages 245-250, ISSN 0747-5632.

[13] Rodríguez, J. M., Merlino, H., \& Fernández, E. (2014). Comportamiento Adaptable de Chatbots Dependiente del Contexto. Revista Latinoamericana de Ingenieria de Software, 2(2), 115-136.

[14] Rodríguez, Juan, Merlino, Hemán y Fernández, Enrique. Comportamiento Adaptable de Chatbots Dependientes del Contexto. Facultad de Ingeniería, Universidad Nacional de Lanús. Argentina : s.n., 2014

[15] Gianvecchio, S., Xie, M., Wu, Z., \& Wang, H. (2011). Humans and bots in internet chat: measurement, analysis, and automated classification. IEEE/ACM Transactions on Networking (TON), 19(5), 1557-1571.

[16] Tur, G., \& Mori, R. D. (2011). Spoken language understanding: systems for extracting semantic information from speech. Hoboken, NJ: Wiley.

[17] Stuart Rusell, Peter Norving 2003. Inteligencia Artificial, Un enfoque Moderno. Editorial Prentice Hall.

[18] Sidorov, G., Ibarra Romero, M., Markov, I., GuzmanCabrera, R., Chanona-Hernández, L., \& Velásquez, F. (2016). Detección automática de similitud entre programas del lenguaje de programación Karel basada en técnicas de procesamiento de lenguaje natural. Computación y Sistemas, 20(2), 279-288.

[19] D. Namiot and M. Sneps-Sneppe, "On iot programming," International Journal of Open Information Technologies, vol. 2, no. 10, 2014.

[20] J. Zhou, R.Q. Hu and Y. Qian. "Scalable Distributed Communication Architectures to Support Advanced Metering Infrastructure in Smart Grid". Parallel Distrib. Syst. IEEE Trans. Vol. $23 \mathrm{~N}^{\circ}$ 9, pp. 1632-1642. 2012. ISSN: 10459219. DOI:10.1109/TPDS.2012.53.

[21] .J. Wu, Y.F. Kao and Y.C. Tseng. "From Wireless Sensor Networks Towards Cyber Physical Systems". Pervasive Mob. Comput. Vol. $7 \mathrm{~N}^{\circ} 4$, pp. 397-413. 2011. ISSN: 15741192. DOI: 10.1016/j.pmcj.2011.03.003.

[22] P. Li, S. Guo, S. Member and Z. Cheng. "Joint Optimization of Electricity and Communication Cost for Meter Data Collection in Smart Grid". Emerg. Top. Comput. IEEE Trans. Vol. $1 \mathrm{~N}^{\circ}$ 2, pp. 297 -306. 2013. ISSN: 21686750. DOI:10.1109/TETC.2013.2273890. 